\title{
The Role of Fenestration of the Lamina Terminalis on Symptomatic Vasospasm After Aneurysmal Subarachnoid Hemorrhage: A Clinical Research
}

\author{
Sahika Liva CENGIZ¹, Mustafa Kemal ILIK², Fatih ERDI ${ }^{3}$, Mehmet Erkan USTUN \\ 'Serik State Hospital, Department of Neurosurgery, Antalya, Turkey \\ ${ }^{2}$ Farabi Hospital, Department of Neurosurgery, Konya, Turkey \\ ${ }^{3}$ Necmettin Erbakan University, Meram Faculty of Medicine, Department of Neurosurgery, Konya, Turkey \\ ${ }^{4}$ Natomed Hospital, Department of Neurosurgery, Ankara, Turkey
}

\section{ABSTRACT}

AIM: To compare the beneficial effects of cisternal blood clot evacuation with or without microsurgical fenestration of the lamina terminalis (LT) on symptomatic vasospasm during the course of aneurysmal subarachnoid hemorrhage.

MATERIAL and METHODS: This was a clinical, retrospective study that included 72 patients with aneurysmal subarachnoid hemorrhage (aSAH). The patient group that underwent aneurysm clipping with only extensive cisternal drainage of the subarachnoid blood clot was defined as the non fenestrated lamina terminalis group (NonFLT group, $n=39$ ). The patient group that underwent aneurysm clipping with extensive cisternal drainage of the subarachnoid blood clot combined with microsurgical fenestration of LT was defined as the fenestrated lamina terminalis group (FLT group, $n=33$ ).

All patients were operated by the same experienced neurovascular surgeon, the senior author of this article (MEU), and his colleagues. New cerebral infarct development was determined with computed tomography, Glasgow Outcome Score (GOS), clinical vasospasm rate and Hunt-Hess scale $(\mathrm{H} \& \mathrm{H})$ grade before and after surgery and duration of hospital stay of the patients were assessed and compared.

RESULTS: A favorable clinical outcome was obtained from $51.2 \%$ of the NonFLT group patients and $72.66 \%$ of the FLT group patients. And FLT group patients have better GOS and H\&H grade. Clinical vasospasm rate and duration of hospital stay were also significantly reduced in FLT group patients.

CONCLUSION: Wide fenestration of lamina terminalis as a cerebrospinal fluid diversion technique may be very beneficial in the surgical treatment of aneurysmal SAH.

KEYWORDS: Cisternal blood clot, Lamina terminalis, Subarachnoid hemorrhage, Symptomatic vasospasm

\section{INTRODUCTION}

$\longrightarrow$ erebral vasospasm as a most frequent complication of aneurysmal subarachnoid hemorrhage (aSAH) occurs in $70 \%$ of patients and cause significant mortality and morbidity $(2,13)$. Angiographic evidence of vasospasm is apparent in $70 \%$ of patients, while clinical manifestation of vaso- spasm is present in one third of patients $(8,10,11,19)$. Symptomatic vasospasm is more critical, even after the aneurysm has been treated. Vasospasm is a multi-factorial and multistage process. Although thick subarachnoid blood is the principal precipitating factor, the exact cause of arterial narrowing after aSAH is poorly understood yet. Various treatment strategies including antifibrinolytic therapy, routine cerebrospinal 
fluid (CSF) diversion, intraoperative hypothermia, neuroprotective drugs, intraoperative angiography and decompressive hemicraniectomy have been used for the treatment of this severe complication but none of them were found highly effective (15). Different CSF diversion techniques include ventricular or spinal drains and lamina terminalis (LT) fenestration have been used for prevention and treatment of clinical vasospasm (15). In this retrospective study we observe the possible beneficial effects of wide (at least $1 \mathrm{~cm}$ ) LT fenestration in our surgically treated high grade aSAH patients.

\section{MATERIAL and METHODS}

\section{Study Description}

This is a clinical retrospective study which based on the medical records of 72 consecutive patients with aSAH admitted to Konya Necmettin Erbakan University, Meram Faculty of Medicine between January 2005 and January 2012.

All patients were operated within the acute phase of aSAH (within 3 days) and underwent clipping of ruptured aneurysm by a single experienced vascular neurosurgeon, senior author (MEU) and his colleagues as other authors.

The patients were divided into two groups. The patient group that underwent aneurysm clipping with only extensive cisternal drainage of the subarachnoid blood clot was defined as the Non fenestrated lamina terminalis group (NonFLT group, $\mathrm{n}=39$ ). The patient group that underwent aneurysm clipping with extensive cisternal drainage of the subarachnoid blood clot combined with microsurgical fenestration of LT was defined as the Fenestrated lamina terminalis group (FLT group, $\mathrm{n}=33$ ).

\section{Diagnosis of SAH}

The diagnosis of SAH was established on the basis of admission computed tomography (CT) scans. All patients had positive CT scans and none of them underwent lumbar puncture for diagnosis or CSF analyse. Before surgery all patients underwent cerebral angiography with or without CT angiography. CT angiography was performed to detect the relation of aneurysm with bony structures.

\section{SAH Grading}

Clinical condition of the patients at admission was recorded by using the Hunt and Hess (H\&H) SAH grading scale (1). CT scans of the patients at admission were recorded and graded by using Fisher scale (22).

\section{Surgical Method}

During surgery, the carotid and optic cisterns were widely opened in both groups. Cleaning of the blood clot from ipsilateral sylvian fissure and carotid cistern was performed. Gyrus rectus resection was not routinely performed and reserved for aneurysms which we could not completely expose after a routine approach to the anterior communicating artery complex.

In the NonFLT group, drainage of CSF by suction and meticulous cisternal cleaning of the subarachnoid clot to the maximum extent was performed before clipping. In the FLT group, after opening the carotid and optic cisterns, dissection is further continued along the ipsilateral optic nerve, followed by gentle retraction of the ipsilateral frontal lobe to reach the lamina terminalis. At least $1 \mathrm{~cm}$ longitudinal incision along the midline avascular zone of the LT was performed with a no:11 scalpel blade after protection of the adjacent important neurovascular structures. Patency of the perforation was verified by a significant outpouring of CSF (1). This was followed by opening of Liliequist membrane and clot removal from the interpeduncular cistern. A Gelfoam ${ }^{\circledR}$ pledget soaked with a solution of $3 \%$ papaverine was placed over the clipped aneurysm at least 30 minutes before surgical closure of anatomical layers in both groups.

\section{Exclusion Criteria}

Exclusion criteria were determined as: Patients had low Fisher grade (Fisher grade 1-2). The patients had SAH due to traumatic origin or other reasons such as arteriovenous malformation or hematological and coagulation disorders.

\section{Inclusion Criteria}

Patients suffering SAH due to a ruptured aneurysm and developing symptoms of vasospasm after surgery were considered for inclusion in our study. Symptomatic vasospasm was defined as: New onset of focal neurological deficiency or impairment in the cognitive functions after exclusion of other probable reason of deterioration such as rebleeding, hydrocephalus, surgery related complications, brain edema, electrolyte imbalance, infection, or convulsion (6). Because the rates of post-SAH cerebral vasospasm largely relate to the amount of blood in the subarachnoid spaces, patients with Fisher $\geq 3$ who are at particular risk were included in the study.

All patients underwent early surgery within 72 hours. Among 72 patients the coexistence of hydrocephalus with cerebral vasospasm was not detected at admission. Clinical factors such as demographic variables; age, sex, social history, alcohol or cigarette consumption, previous medical history (diabetes mellitus, hypertension, cardiac disturbance, cerebrovascular stroke or headache), clinical and laboratory properties at onset symptoms on SAH, mean arterial pressure on admission, were recorded (Table I). The rate of clinical vasospasm was established by the number of patients who developed symptomatic vasospasm after surgical intervention divided by the total number of patients (4). Admission and all follow-up CT scans were independently evaluated by a study neurosurgeon for the amount and location of blood, and the presence of infarction. Angiographic findings and results were also recorded. Evidence of arterial spasm by angiography was generally used to support the diagnosis of cerebral vasospasm but not in all patients.

The localization of the ruptured aneurysm was evaluated by same neurosurgeon in all patients. After the surgery, standard medical management was used, including administration of nimodipine, volume expansion, and maintenance of optimal general hemodynamic and medical status. In patients whom cerebral vasospasm developed, hypertensive-hypervolemic therapy $(\mathrm{HHT})$ to preserve systolic blood pressure at $>200$ 
$\mathrm{mm} \mathrm{Hg}$ was used as described (24). When significant clinical symptoms continued notwithstanding HHT, CT angiography was performed for further investigation.

\section{STATISTICAL ANALYSIS}

The Student t-test (parametric), Mann-Whitney test (nonparametric) and Chi-square test for comparison of proportions and multivariate analysis were used. A p value of $<0.05$ was accepted as significant.

\section{RESULTS}

\section{Patient Characteristics}

A summary of patient characteristics is presented in Table I. Among 72 patients, no statistically significant difference was found regarding admission $\mathrm{H} \& \mathrm{H}$ grade, age, sex, Fisher grade, time of surgery, and aneurysm location.

Mean ages were $49.8 \pm 12.10$ year in the NonFLT group and $48.3 \pm 8.64$ year in the FLT group. In the NonFLT group and FLT group, the female-to-male ratio was 2.25 and 1.1 respectively.

All patients had only one ruptured aneurysm. The location of the ruptured aneurysm was determined as 27 middle cerebral artery, 19 anterior communicating artery, 9 anterior cerebral artery, 7 posterior communicating artery, 4 clinoidal segment, 4 ophthalmic artery and 2 aneurysms were located at the bifurcation of internal carotid artery (Table II).

\section{LT Fenestration and Development of Cerebral Vasospasm}

The rate of clinical vasospasm was established by the number of patients who developed symptomatic vasospasm after surgical intervention divided by the total number of patients (23.61\%). Clinical cerebral vasospasm occurred in 2 patients $(6.06 \%)$ in the FLT group and 15 patients $(38.4 \%)$ in the NonFLT group $(p<0.05)$. In the FLT group, one of two patients who had cerebral vasospasm showed severe brain edema and hematoma in the third ventricle on postoperative 7 th day CT imaging. The patient was re-operated and hematoma was evacuated and the patient recovered within two days.

The 17 patients (23.61\%) who developed clinical cerebral vasospasm underwent standard medical management, including administration of nimodipine, volume expansion, and maintenance of optimal general hemodynamic and medical status.

Mean hospital stay was $26 \pm 4.2$ days in the NonFLT group and $14 \pm 2.7$ days in the FLT group. When compared with the NonFLT group, the FLT group had a significant shorter hospital stay $(\mathrm{p}<0.05)$.

Follow-up examinations were performed after 12 to 180 days in postoperative period. The mean follow- up time was 95 days in the NonFLT group and 78 days in the FLT group.

No patients were lost during the follow up period. A favorable clinical outcome was obtained in $51.2 \%$ of the NonFLT group patients and $72.66 \%$ of the FLT group patients. No procedure-related complications were observed in the FLT group patients.

$\mathrm{H} \& \mathrm{H}$ grades of the patients were recorded before the surgery and before the discharge/death of the patient (after surgery).

In the NonFLT group, H\&H grades changed statically insignificantly after the surgery ( $p>0.05)$. According to GOS scores,

Table I: Demographic Characteristics of the Patients

\begin{tabular}{|c|c|c|c|}
\hline & NonFLT & FLT & $P$ value \\
\hline Mean Age (year) & $49.8 \pm 12.109$ & $48.3 \pm 8.64$ & $(p>0.05)$ \\
\hline MEABP preoperatively & $13.6 \pm 2.4 \mathrm{mmHg}$ & $13.4 \pm 2.7 \mathrm{mmHg}$ & $(p>0.05)$ \\
\hline MEABP postoperatively & $16.4 \pm 2.9 \mathrm{mmHg}$ & $15.8 \pm 2.6 \mathrm{mmHg}$ & $(P>0.05)$ \\
\hline Smoking Habbit & 2 & 3 & \\
\hline Alcohol Usage & None & None & \\
\hline Past Illness History & $\begin{array}{c}3 \\
2 \text { Diabetes Mellitus } \\
1 \text { Chronic Obstructive Pulmonary } \\
\text { Disease }\end{array}$ & $\begin{array}{c}5 \\
3 \text { Diabetes Mellitus } \\
1 \text { Chronic Obstructive Pulmonary } \\
\text { Disease } \\
\text { 1 Prostate CA }\end{array}$ & \\
\hline Fisher SAH Grade & $\begin{array}{c}3^{\star} \\
3.30 \pm 0.39^{\varepsilon}\end{array}$ & $\begin{array}{c}3^{*} \\
3.34 \pm 0.42^{\varepsilon}\end{array}$ & $\begin{array}{l}(p>0.05) \\
(p=0.14)\end{array}$ \\
\hline Day of Aneurysm Surgery & 1.8 & 1.7 & $P=0.1$ \\
\hline Gender & $\begin{array}{l}27 \text { Female } \\
12 \text { Male }\end{array}$ & $\begin{array}{l}17 \text { Female } \\
16 \text { Male }\end{array}$ & \\
\hline
\end{tabular}

*Median (Minimum: 3-Maximum: 4), ${ }^{\boldsymbol{E}}$ Mean \pm SD, SAH: Subarachnoid hemorrhage, MEABP: Mean arterial blood pressure. 
Table II: Localization of the Ruptured Aneurysms in Each Group

\begin{tabular}{lccccccc}
\hline Group & MCA & AcoA & $\begin{array}{c}\text { Clinoidal Seg- } \\
\text { ment }\end{array}$ & $\begin{array}{c}\text { Ophthalmic } \\
\text { Segment }\end{array}$ & ACA & $\begin{array}{c}\text { ICA } \\
\text { bifurcation }\end{array}$ \\
\hline Non FLT & 18 & 10 & 1 & 1 & 9 & - \\
\hline FLT group & 9 & 9 & 3 & 3 & - & 7 & - \\
\hline
\end{tabular}

MCA: Middle cerebral artery, AcoA: Anterior communicating artery, ACA: Anterior cerebral artery, PcoA: Posterior communicating artery, ICA: Internal carotid artery.

Table III: Overall Results of the Study

\begin{tabular}{|c|c|c|c|}
\hline & NonFLT & FLT & $P$ value \\
\hline H\&H SAH Grade (Before Surgery) & $\begin{array}{c}3^{\star} \\
3.10 \pm 0.64^{£}\end{array}$ & $\begin{array}{c}4^{\star} \\
3.27 \pm 0.8^{\varepsilon}\end{array}$ & $\begin{array}{l}p>0.05 \\
p>0.05\end{array}$ \\
\hline H\&H SAH Grade (After Surgery) & $\begin{array}{c}4.5^{\star} \\
3.76 \pm 0.52^{£}\end{array}$ & $\begin{array}{c}3^{\star} \\
3.01 \pm 026^{£}\end{array}$ & $\begin{array}{l}p=0.037 \\
p<0.05\end{array}$ \\
\hline GOS & $\begin{array}{c}3^{*} \\
3.27 \pm 0.68^{\varepsilon}\end{array}$ & $\begin{array}{c}4.5^{\star} \\
4.12 \pm 0.44^{£}\end{array}$ & $\begin{array}{l}p<0.05 \\
p=0.033\end{array}$ \\
\hline Hospital Stay (Day) & $26 \pm 4.2^{\varepsilon}$ & $14 \pm 2.7^{\varepsilon}$ & $\mathrm{p}<0.05$ \\
\hline Favorable Clinical Outcome & $51.2 \%$ & $72.66 \%$ & $\mathrm{p}<0.05$ \\
\hline
\end{tabular}

*Median (Minimum:1 - Maximum: 5), ${ }^{\mathfrak{E}}$ Mean $\pm S D$, H\&H SAH Grade: Hunt and Hess SAH grade, GOS: Glasgow outcome score.

clinical status showed excellent improvement in 16 patients (41.0\%), 4 patients showed mild improvement (\%10.2), were worse in 11 patients $(28.3 \%)$ and remained unchanged in 8 (20.5\%) patients.

In the FLT group, H\&H scores were changed statically significantly after surgery $(p=0.008)$. According to GOS scores, 22 (66.6\%) FLT group patients showed excellent improvement, 2 patients in this group $(6.06 \%)$ showed mild improvement. Clinical status remained unchanged in 9 (27.2\%) patients.

Among the Non FLT group patients, 4 patients (10.2\%) who developed cerebral vasospasm died within 5-17 days after surgery.

The statistically calculated favorable efficiency values were $51.2 \%$ in the NonFLT group versus $72.66 \%$ in the FLT group. The statistical relative efficiency ratio of the study was $4.27 \%$.

The results of the study are briefly summarized in Table III.

\section{DISCUSSION}

Vasospasm resulting from aSAH is a complicated multifactorial and multistage process. Although thick subarachnoid blood is the principal precipitating factor, the exact cause of arterial narrowing after aSAH is poorly understood. The arterial narrowing that occurs in cerebral vasospasm is typically a transient or temporary event, naturally lasting from a few days up to 3 weeks (11). Despite the reversible nature of this condition, its occurrence may still be harmful or even fatal. Thus vasospasm remains the major leading factor of fatal course and poor neurological outcome $(8,11,15,19)$. Currently aneurysm clipping is still considered as first line treatment and radical cisternal blood evacuation is a well-recognized policy. In surgical treatment of ruptured aneurysm the radical cleaning of large amounts of cisternal subarachnoid blood and low incidence of symptomatic vasospasm are well described $(1,9,22)$. By meticulous surgical aspiration of blood clots in basal cisterns, many authors have substantially reduced the rates of symptomatic and angiographic vasospasm $(7,12,16$ 18,20,21,23).

During aneurysm surgery, CSF drainage is essential for avoiding aggressive retraction of the brain and for reducing the intracranial pressure (ICP) $(1,7,9,15,22)$. The main methods for CSF diversion techniques are opening the basal cisterns, fenestration of the LT, external ventricular drainage, and lumbar drainage $(12,16-18,20,21)$.

The idea of creation of an alternate path for the redirection of CSF from the ventricular system into the subarachnoid space was first described by Dandy in $1922(5,15)$. The original Dandy technique was modified during the later years, and fenestration of the LT become an adjunctive maneuver for avoiding SAH-related hydrocephalus and vasospasm $(13,15)$. Yasargil also reported LT fenestration as a part of his surgical strategy for ruptured anterior circulation aneurysms (23).

The relationship between LT fenestration and the incidence of shunt dependent hydrocephalus after aSAH was first described by Komotar et al. (14). In their retrospective study, they found the rate of shunt-dependent hydrocephalus in the fenestration cohort as $2.3 \%$ versus a shunt rate of $12.6 \%$ for all other patients $(P=0.011$; odds ratio, 0.15$)$. They stated that LT fenestration provided $>80 \%$ protection from this serious complication (14). 
Andaluz and Zuccarello (1) investigated the effects of LT fenestration on hydrocephalus, vasospasm, and frontobasal injury in their prospective study on 106 patients with anterior communicating artery aneurysms and Fisher Grade III aSAH. Patients were divided into fenestration or nonfenestration groups based on surgeon preference, with 53 patients in each group. They also reported $70 \%$ less shunt-dependent hydrocephalus in patients undergoing fenestration compared with those who did not in accordance with the previous study from Komotar et al. (14). In addition, the incidence of vasospasm was also reduced after fenestration of the LT, in the LT fenestrated group. Overall outcome was noted to improve significantly in those patients undergoing fenestration of the $\mathrm{LT}(1,14)$.

It was suggested that, the decrease in the concentration of blood-derived spasmogenic agents in the CSF by LT fenestration could alter the incidence of vasospasm $(1,15)$. The current literature supports our findings with more favorable outcomes by significantly decreased shunting rate and vasospasm incidence by LT fenestration (15).

We suggest that the perforation width of the lamina terminalis is crucial. To avoid risks such as early closure of the fenestration area due to cerebral edema or hematoma, we make an incision at least $1 \mathrm{~cm}$ long. By opening this anatomical structure, rapid and effective CSF removal can be achieved and we also protect the frontal lobe from aggressive retraction during surgical dissection of the aneurysm.

Our study also has some limitations. First of all, it includes relatively small sample size and follow-up time. Also we know transcranial Doppler measurements and post-surgery cerebral angiography evaluation would be more satisfactory for comparing the overall results. Post-surgery cerebral angiography to confirm a diagnosis of vasospasm was not performed in all patients, because its clinical utility in patients with vasospasm remains controversial and this procedure has significant complications. It was concluded that the risk/ benefit ratio does not warrant serial angiography evaluation (3). Moreover clinical vasospasm can be detected by a physician on physical examination of a patient, regardless of the angiographic findings.

\section{CONCLUSION}

According to the results of our study, wide fenestration of lamina terminalis as a cerebrospinal fluid diversion technique may be very beneficial in the surgical treatment of aneurysmal $\mathrm{SAH}$. Further multicenter randomized controlled studies on a large number of patients may be more useful for determining the exact benefits or complications of this surgical strategy.

\section{REFERENCES}

1. Andaluz N, Zuccarello M: Fenestration of the lamina terminalis as a valuable adjunct in aneurysm surgery. Neurosurgery 55:1050-1059,2004

2. Barnett H, Stein B, Mohr J, Yatsu F: Stroke; Pathophysiology, diagnosis, and management. Vol: 1. New York: Churchill Livingstone, 1986
3. Bruneau M, Rynkowski M, Smida-Rynkowska K, Brotchi J, De Witte O, Lubicz B: Long-term follow-up survey reveals a high yield, up to $30 \%$ of patients presenting newly detected aneurysms more than 10 years after ruptured intracranial aneurysms clipping. Neurosurg Rev 34:485-496, 2011

4. Claassen J, Bernardini GL, Kreiter K, Bates J, Du YE, Copeland D, Connolly ES, Mayer SA: Effect of cisternal and ventricular blood on risk of delayed cerebral ischemia after subarachnoid hemorrhage: The Fisher scale revisited. Stroke 32:2012-2020, 2001

5. Dandy W: An operative procedure for hydrocephalus. Johns Hopkins Hosp Bull 33:189, 1922

6. Frontera JA, Fernandez A, Schmidt JM, Claassen J, Wartenberg KE, Badjatia N, Cannolly ES: Defining vasospasm after subarachnoid hemorrhage: What is the most clinically relevant definition? Stroke 40:1963-1968, 2009

7. Haque R, Kellner CP, Komotar RJ, Connolly ES, Lavine SD, Solomon RA, Meyers PM: Mechanical treatment of vasospasm. Neurol Res 31:638-643,2009

8. Hop JW, Rinkel GJ, Algra A, Van Gijn J: Initial loss of consciousness and risk of delayed cerebral ischemia after aneurysmal subarachnoid hemorrhage. Stroke 30:22682271,1999

9. Kaku Y, Yamashita K, Kokuzawa J, Hatsuda N, Andoh T: Treatment of ruptured cerebral aneurysms - clip and coil, not clip versus coil. Acta Neurochir Suppl 107:9-13,2010

10. Kim SS, Park DH, Lim DJ, Kang SH, Cho TH, Chung YG: Angiographic features and clinical outcomes of intraarterial nimodipine injection in patients with subarachnoid hemorrhage-induced vasospasm. J Korean Neurosurg Soc 52:172-178, 2012

11. Kocaogullar $\mathrm{Y}$, Ilik K, Esen H, Koc O, Guney O: Preventive effects of intraperitoneal selenium on cerebral vasospasm in experimental subarachnoid hemorrhage. J Neurosurg Anesthesiol 22:53-58, 2010

12. Kodama N, Matsumoto $M$, Sasaki T, Konno $Y$, Sato $T$ : Cisternal irrigation therapy with urokinase and ascorbic acid for prevention of vasospasm. Acta Neurochir Suppl 77:171174, 2001

13. Komotar RJ, Hahn DK, Kim GH, Khandji J, Mocco J, Mayer SA, Connolly ES Jr: The impact of microsurgical fenestration of the lamina terminalis on shunt-dependent hydrocephalus and vasospasm after aneurysmal subarachnoid hemorrhage. Neurosurgery 62:123-132,2008

14. Komotar RJ, Olivi A, Rigamonti D, Tamargo RJ: Microsurgical fenestration of the lamina terminalis reduces the incidence of shunt-dependent hydrocephalus after aneurysmal subarachnoid hemorrhage. Neurosurgery 51:1403-1413, 2002

15. Komotar RJ, Zacharia BE, Mocco J, Connoly ES Jr: Controversies in the surgical treatment of ruptured intracranial aneurysms: The First Annual J. Lawrence Pool Memorial Research Symposium controversies in the management of cerebral aneurysms. Neurosurgery 62: 396-407, 2008

16. Krayenbühl N, Erdem E, Oinas M, Krisht AF: Symptomatic and silent ischemia associated with microsurgical clipping of intracranial aneurysms: Evaluation with diffusion-weighted MRI. Stroke 40:129-133,2009 
17. Lehto $H$, Dashti $R$, Karataş A, Niemelä $M$, Hernesniemi JA: Third ventriculostomy through the fenestrated lamina terminalis during microneurosurgical clipping of intracranial aneurysms: An alternative to conventional ventriculostomy. Neurosurgery 64:430-434,2009

18. Proust F, Hannequin D, Langlois O, Freger P, Creissard P: Causes of morbidity and mortality after ruptured aneurysm surgery in a series of 230 patients. The importance of control angiography. Stroke 26:1553-1557, 1995

19. Qureshi Al, Sung GY, Razumovsky AY, Lane K, Straw RN, Ulatowski JA: Early identification of patients at risk for symptomatic vasospasm after aneurysmal subarachnoid hemorrhage. Crit Care Med 28: 984-990,2000

20. Sakaki S, Ohta S, Kuwabara H, Shiraishi M: The role of ventricular and cisternal drainage in the early operation for ruptured intracranial aneurysms. Acta Neurochir 88:87-94, 1987
21. Sasaki T, Kodama N, Kawakami M, Sato M, Asari J, Sakurai Y, Watanabe K, Onuma T, Matsuda T: Urokinase cisternal irrigation therapy for prevention of symptomatic vasospasm after aneurysmal subarachnoid hemorrhage: A study of urokinase concentration and the fibrinolytic system. Stroke 31:1256-1262, 2000

22. Smith ML, Abrahams JM, Chandela S, Smith MJ, Hurst RW, Le Roux PD: Subarachnoid hemorrhage on computed tomography scanning and the development of cerebral vasospasm: The Fisher grade revisited. Surg Neurol 63:229234, 2005

23. Yasargil MG: Microsurgical anatomic features of the lamina terminalis. Neurosurgery 50:569-577, 2002

24. Zacharia BE, Ducruet AF, Hickman ZL, Grobelny BT, Fernandez L, Schmidt JM, Narula R, Ko LN: Renal dysfunction as an independent predictor of outcome after aneurysmal subarachnoid hemorrhage: A single-center cohort study. Stroke 40:2375-2381, 2009 